Can Participants' Characteristics Predict Benefit from a Multimodal Burnout Prevention Program? Secondary Analysis of a Randomized Controlled Trial conducted in Germany

Anushiya C. Vanajan ${ }^{\mathrm{a}, \mathrm{b}}$, Marita Stier-Jarmer ${ }^{\mathrm{c}}$, Ivana Ivandic ${ }^{\mathrm{d}}$, Angela Schuh ${ }^{\mathrm{c}} \&$ Carla Sabariego ${ }^{\mathrm{c}, \mathrm{e}, \mathrm{f}}$

${ }^{a}$ Research group for Work and Retirement, Netherlands Interdisciplinary Demographic Institute, The Hague, The Netherlands

${ }^{\mathrm{b}}$ Community and Occupational Medicine, Department of Health Sciences, University Medical Center Groningen, Groningen, The Netherlands

${ }^{\mathrm{c}}$ Chair for Public Health and Health Services Research, Institute for Medical Information Processing, Biometry, and Epidemiology, Ludwig Maximilians University, Germany

${ }^{\mathrm{d}}$ Institute and Outpatient Clinic for Occupational, Social, and Environmental Medicine, University Hospital Munich, Ludwig Maximilians University, Munich, Germany

${ }^{\mathrm{e}}$ Department of Health Sciences and Health Policy, University of Lucerne, Switzerland ${ }^{\mathrm{f}}$ Swiss Paraplegic Research, Switzerland 
Final Author's Version

\title{
Can Participants' Characteristics Predict Benefit from a Multimodal Burnout Prevention Program? Secondary Analysis of a Randomized Controlled Trial conducted in Germany
}

\begin{abstract}
Chronic psychological distress appears to be increasing markedly among the working population. A recent randomized controlled trial (RCT) supported the effectiveness of a threeweek outpatient burnout prevention program - comprised of stress management interventions, relaxation, physical exercise and moor baths followed by massage - in reducing perceived stress and emotional exhaustion. However, the effectiveness of treatments in the real-world that were shown to be efficacious in RCTs is related to the appropriate selection of individuals who are most likely to yield sustainable gains. Therefore, factors predicting the intensity of response and nonresponse of individuals to treatment are of interest. This secondary data analysis aims to explore predictors of response to the outpatient burnout prevention program in a sample of eighty employed persons at high risk of burnout. Hierarchical linear regression was performed to identify predictors of successful response - defined by lower perceived stress at last follow up. Nutritional behavior, symptoms of eating disorder syndrome and well-being were significant predictors of perceived stress at last follow up, when adjusted for age, sex, education level, baseline stress values and timing of intervention. Persons with low levels of well-being, poor nutritional behavior and higher symptoms of eating disorders should be given special care and attention to ensure that they respond well to the outpatient burnout prevention program.
\end{abstract}

Keywords: stress, professional burnout, program effectiveness, intervention 


\section{Introduction}

Chronic psychological distress has been increasing markedly among the working population. Psychological distress has recently replaced musculoskeletal diseases as the leading cause of absenteeism and long-term work incapacity in many countries. ${ }^{1-3}$ In Germany, the number of persons on sick leave due to mental disorders resulting from psychological distress nearly doubled from 1994 to 2010 and accounted for $9.1 \%$ of all persons on sick leave. ${ }^{4}$ In addition, in 2011, $12.5 \%$ of the working population in Germany reported experiencing stress in their workplaces. ${ }^{5}$

The majority of psychological distress can be attributed to workplace stress and burnout, both of which are treatable and in some cases preventable. ${ }^{1,2,6}$ Workplace stress is defined as the change in one's physical and mental state as a response to challenging and threatening workplace risk factors. ${ }^{7}$ Burnout is conceptualized to be a long-term reaction to stress, characterized by persistent emotional exhaustion, cynicism and reduced personal accomplishment. ${ }^{8,9}$ A representative survey conducted in 2011 in Germany found that 1.9 million people aged 14 years and older had been diagnosed with burnout syndrome at least once in their lives. ${ }^{10}$

Workplace stress and burnout account for significant psychological, physiological and economical costs on both individual and organizational level. Burnout was found to increase the risk of future illness and therein certified sick-leave absences. ${ }^{11}$ Employees with high levels of workplace stress and symptoms of burnout are at greater risk for physical disorders such as cardiovascular disease, ${ }^{12,13}$ cancer,${ }^{14}$ diabetes, ${ }^{15}$ musculoskeletal pain, ${ }^{11,16,17}$ respiratory diseases ${ }^{11}$ and impaired digestive functioning, ${ }^{18}$ as well as psychological disorders such as depression, persistent anxiety, fatigue, psychosis, sleep disturbances, impaired memory and social impairments. ${ }^{18-20}$ These negative health consequences further contribute to poor morale, hostility and interpersonal conflict at work. ${ }^{21}$ Consequently, workplaces suffer from high rates 
of absenteeism, presentism, turnover, medical costs, short and long-term worker disability, accidents and lower productivity. ${ }^{22-24}$

In the recent years, Germany experienced a considerable increase in the number of sick leave days and cases of early retirement due to mental disorders. ${ }^{25}$ The number of days off work due to mental disorders increased from 41 million days in 2008 to 79 million days in 2013..$^{26,27}$ Similarly, the proportion of early retirement caused by mental and behavioral disorders (ICD-10 F00-F99) increased from $24.2 \%$ in the year 2000 to $43.1 \%$ by $2014 .{ }^{25}$ In an economical perspective, in Germany, direct (prevention, rehabilitation and treatment) and indirect (lost working years, disability and premature death) costs of job strain, totaled $€ 29.2$ billion annually. ${ }^{28}$

Considering the high human and financial burden of workplace stress, it represents a serious public health concern that needs to be addressed. The past years have witnessed the development of numerous individual focused and organization focused stress management and prevention interventions. These interventions range from individual psychological services and mind-body interventions to restructuring of the organization - most of which appear to be effective at reducing workplace stress. ${ }^{29-31}$

A recent randomized controlled trial (RCT) supported the effectiveness of a novel outpatient burnout prevention program combining traditional outpatient health resort treatments with stress management interventions. ${ }^{6}$ The 3 -week program included stress management interventions, relaxation, physical exercise and moor applications. This program aimed to reduce stress, burnout symptoms, psychological symptoms, back pain and number of sick days, while increasing well-being and health status, among highly stressed participants who were at risk of burnout. Participants experienced statistically significant immediate improvement in all outcome measures, which slightly declined during the first three months post-intervention, but remained stable for at least another three months. ${ }^{6}$ 
Final Author's Version

Although shown to be efficacious in RCTs, the effectiveness of interventions in real life environments largely depends on the appropriate selection of individuals who are most likely to yield sustainable gains. Moreover, poor responders will require additional attention and care to respond positively to the intervention. In order to guide the early identification of good and poor responders, it is important to know which factors predict the intensity of the response. This is of interest not only for health professionals planning and carrying out interventions, but also for health insurance representatives granting the treatment as well. Thus, the objective of this study is to carry out a secondary data analysis of data gathered in the $\mathrm{RCT}^{6}$ to identify predictors of response to the outpatient burnout prevention program in a sample of employed persons at high risk of burnout. Due to the exploratory nature of the study, no hypothesis were developed antecedently.

\section{Methods}

\section{Study design and Setting}

A secondary analysis of a two-arm RCT with pre- and post-intervention (T0, T1) assessment points and follow-ups at 1-, 3-, and 6-months post-intervention, respectively (T2 T4) was conducted. In the original RCT, participants ( $\mathrm{N}=88)$ were randomly assigned in a 1:1 ratio using permuted blocks of 10 to the immediate intervention group (IGG) or the wait-list control group (WG), the latter of whom received the intervention 6 months after study enrollment. This secondary analysis compiled data obtained from the IGG and WG, thus creating a combined dataset for analyses. The original study, conducted in 2014 in Bavaria, Germany, was approved by the ethics committee of Ludwig Maximilians University, Munich. Refer here for the original study. ${ }^{6}$ 
Final Author's Version

\section{Participants}

The original study aimed to recruit $n=90$ participants based on sample size estimation presuming an effect size of 0.35 , a power of 0.8 , a significance level of 0.05 and a dropout rate of $10 \%$. Interested persons were recruited using print and electronic advertisements between December 2013 and February 2014. After recruitment, participants were invited to complete two screening questionnaires: The Maslach Burnout Inventory-General Survey (MBI-GS-D) ${ }^{32}$ and the Perceived Stress Questionnaire (PSQ). ${ }^{33,34}$ Based on results of the screening, only interested persons with above-average levels of stress and an increased risk of developing a burnout syndrome were included in the RCT. For instance, we only included interested persons who were at risk of burnout or incipient burnout defined by scores between 3.6 and 5.2 on the Emotional Exhaustion Scale (MBI-EE) of the MBI-GS-D. These scores were derived from unpublished work as MBI-GS-D cutoff scores specific to the German population is not available. ${ }^{35-37}$ Moreover, interested persons also had to show above-average levels of perceived stress indicated by a total PSQ score equal to or over 50 which corresponds to the mean plus one standard deviation in healthy adults. ${ }^{34}$ Additionally, we chose to recruit participants who were between the ages of $18-70$ years, had adequate fitness and general health status and were insured with a specific health insurance (Barmer GEK) or were self-paying patients. Participants were not compensated for enrolling into the study. However, they received compensation for filling out the questionnaire. The sample for this secondary data analysis consisted of the original 88 participants but excluded 8 participants from the WG who completed baseline measures but did not participate in the intervention. This produced a final sample of $\mathrm{N}=80$.

\section{Intervention}

The 3-week prevention program focused on four key therapeutic elements: 
1. Stress management interventions (SMI) on burnout prevention (10 two-hour sessions, in groups of 8-12 participants),

2. Relaxation techniques: Hatha-Yoga (5 one-hour sessions), Qigong (5 one-hour sessions), mindfulness training (10 twenty-minute sessions) and progressive muscle relaxation (6 one-hour sessions),

3. Physical exercise: Back school (7 one-hour sessions) and endurance sports activities (7 one-hour sessions), and

4. Moor applications ( $7 \mathrm{x}$ full moor baths $\left(42^{\circ} \mathrm{C}, 20\right.$ minutes each) $)$ followed by a resting period and massage (20 minutes each).

The SMI was based on the group therapy program for burnout developed for inpatients of the Psychosomatic Clinic in Windach, Germany. The program was modified for prevention purposes and was directed by two experienced psychologists. It mainly encompassed a psychoeducational approach combined with exercises in mindfulness-based therapy, stress management, self-care, goal setting and problem solving. The moor applications were conducted in two local rehabilitation clinics and one spa treatment center. A moor bath is prepared using moor mud (peat pulp) consisting of organic matter, minerals and water. It is widely used in European health resorts as part of balneotherapy to treat a multitude of health complaints. Please refer to Stier-Jarmer et al. $^{6}$ for further information on therapeutic elements and on the ethical and legal framework of the intervention.

\section{Outcomes and Measures}

The primary outcome of this secondary analysis was perceived stress at the last follow up (T4), measured using the revised 20-item German version of the PSQ. ${ }^{33,34}$ The PSQ evaluates participant's subjective experience of stress reactions and perceived external stressors. All items were rated on a 4-point Likert scale ranging from $1=$ "almost never" to 4 
$=$ "usually". Raw scores ranged from 20 to 80 . The raw scores were subsequently transformed into a scale ranging from 0 to 100 , with higher total PSQ scores depicting higher levels of perceived stress. Reliability analysis based on our sample described the PSQ to have high internal consistency (Cronbach's alpha $=0.95)$.

4.1 Control variables. Age, sex, education level, perceived stress at baseline and timing of intervention were used as control variables. The education level variable was recoded into a dichotomous variable ('up to high school education' versus 'university degree or other higher education'). To account for any effects of the randomization arms, for instance seasonality, we controlled for intervention group. Based on when the IGG and WG received the intervention, we referred to IGG as the Spring group and WG as the Autumn group.

4.2 Predictor variables. a) Well-being was measured using the World Health Organization's Well-Being Index (WHO-5). The WHO-5 consists of five positively phrased items that measure individual's well-being over the last two weeks. The items were scored on a 6-point Likert scale ranging from $0=$ "at no time" to $5=$ "all the time". Thereafter, the raw scores were transformed into a scale from 0 to 100 , with higher values indicating better wellbeing. The WHO-5 showed high internal consistency (Cronbach's alpha $=0.92$ ).

b) The total scores of the three subdomains of the MBI-GS-D ${ }^{32}$ : Emotional Exhaustion (EE) (5 items), Cynicism (CN) (5 items), and Professional Efficacy (PE) (6 items), were treated individually as three continuous variables. All items were written as personal statements or attitudes, and rated by the participant on a 7-point Likert scale from $1=$ "never" to $6=$ "every day". Scores for each subscale were derived using the mean which ranged between 1 and 6 , with higher scores indicating increased EE, CN and PE. Scores were created based on original scoring instructions. ${ }^{35}$ Reliability analyses revealed all subscales to have high internal consistencies (EE Cronbach's alpha $=0.86, \mathrm{CN}$ Cronbach's alpha $=0.85, \mathrm{PE}$ Cronbach's alpha $=0.84)$. 
c) Psychiatric symptoms were measured using the 29-item ICD-10 Symptom Rating Scale (ISR). ${ }^{38}$ The items of ISR represents a broad spectrum of psychological symptoms which were rated by their severity on a 5-point Likert scale ranging from $0=$ "does not apply" to $4=$ "applies extremely". The scale provided a total ISR score and syndrome-specific scores for depression, anxiety, obsessive-compulsive disorder, somatoform syndrome and eating disorders. All scales were estimated by their means, following original scoring instructions, ${ }^{39}$ with higher mean scores representing increased symptoms. The ISR demonstrated a high degree of internal consistency (Cronbach's alpha $=0.90)$.

d) Health-related behavior were analyzed using a questionnaire developed by the German Federal Association of Health Insurance Funds for evaluating health courses. ${ }^{40}$ The 12-item questionnaire covers three subtopics: physical behavior, nutritional behavior and stress management, with four items each, scored on a 5-point Likert scale from $1=$ "always" to $5=$ "never". Raw scores ranged from 4 to 20 for each subscale, with higher scores indicating poorer physical behavior, nutritional behavior and stress management. The complete scale of health behavior demonstrated high internal consistency (Cronbach's alpha $=0.84$ ).

\section{Statistical Analysis}

Means, standard deviations (SD) and frequencies were estimated. Hierarchical linear regression analyses were performed to identify predictors of successful response to the outpatient burnout prevention program. Before regression analysis, correlation matrices and tests of multicollinearity were conducted to check model assumptions. Variables with elevated variance inflation factors (VIF), estimated with a VIF over 5.0, were excluded from the analysis. Due to the small sample size, a pre-selection of predictors was conducted using univariate linear regression analyses. Based on univariate linear regression analyses, variables with $\mathrm{p}<0.25$ were subsequently manually included in the hierarchical linear regression. In 
Final Author's Version

order to select the model with the best fit, several hierarchical linear regression models were run simultaneously with varying order combinations of predictor variables. All hierarchical linear regression models were controlled for age, sex, education level, perceived stress at baseline and timing of intervention. The final model was selected based on the Akaike Information Criterion (AIC) (the lower, the better) and founded on the notion that the model should not include more than eight variables, as we abided by the recommendation of a minimum of ten participants to one variable to keep the results stable. Predictors were considered statistically significant when the $\mathrm{p}<0.05$.

To analyze whether missing data were missing completely at random, Little's Missing Completely at Random test was performed. Missing data were then replaced using Multiple Imputation with 5 imputations.

As a sensitivity analysis, we imputed missing values using another method of imputation - Expectation Maximization - to compare the robustness of our results. This sensitivity analysis was based on the recommendations to conduct and compare results after performing different imputation methods and/or listwise deletion. ${ }^{41,42}$ The statistical software, IBM SPSS Statistics for Windows, Version 24.0 (IBM Corp., Armonk, NY, USA) was used.

\section{Results}

Participants $(\mathrm{N}=80$, Spring group $=43)$ were predominantly female $(75.0 \%)$ with a mean age of 50.7 years $(\mathrm{SD}=6.8)$. Most participants were educated up to high school $(61.2 \%)$, were married or cohabiting (62.5\%) and employed on full or part time basis $(70.0 \%)$ (Table 1).

Eleven of the 80 participants $(13.75 \%)$ had missing information, thereby necessitating a method of missing data imputation. Firstly, we checked for the validity of the missing at random assumption using Little's Missing completely at random test. This test established that data were missing completely at random $(\mathrm{p}=1.00)$, thus allowing for multiple imputation. 


\section{Tests of multicollinearity}

Correlations ranged from -0.56 to 0.85 (Supplementary Table 1). VIF remained in the range between 1.13 and 2.11, with acceptable tolerance values (Supplementary Table 2), except for total health behavior at baseline and total psychiatric symptoms at baseline, which were consequently excluded from regression analyses.

\section{Preselection of predictor variables}

All predictor variables, except somatoform syndrome $(\mathrm{p}=0.70)$, were considered in the hierarchical linear regression analysis as their $\mathrm{p}<0.20$.

\section{Predictors of perceived stress at last follow up}

The final regression model was statistically significant $(\mathrm{F}(8,470)=31.18, \mathrm{p}<0.001)$ and accounted for $33.6 \%$ of the total variance of perceived stress (Table 2). Well-being, nutritional behavior and eating disorder syndrome were significant predictors of perceived stress. While higher symptoms of an eating disorder increased perceived stress at last follow up, better scores of well-being and nutritional behavior decreased perceived stress at last follow up. The strongest predictor of perceived stress at last follow-up was having symptoms of an eating disorder.

\section{Sensitivity Analysis}

Similar to the core analysis, correlation matrices, multicollinearity tests and univariate regression analyses led to the exclusion of predictor variables - total health behavior, total psychiatric symptoms and somatoform syndrome - from hierarchical linear regression analyses. In addition, symptoms of anxiety disorder were excluded from the hierarchical linear 
regression as univariate regression analyses revealed that $\mathrm{p}>0.25$. The final regression model was statistically significant $(\mathrm{F}(8,71)=4.71, \mathrm{p}<0.001)$, explaining $27.3 \%$ of the total variance of perceived stress at last follow up (Table 3). Alike the results of the core regression analysis, nutritional behavior and eating disorder syndrome were significant predictors of perceived stress at last follow-up. Higher symptoms of an eating disorder increased perceived stress at last follow-up, while better nutritional behavior decreased perceived stress at last follow-up. Eating disorder syndrome remained the strongest predictor. In contrast to the core analyses, well-being was a nonsignificant predictor of perceived stress at last follow up in the sensitivity analysis.

\section{Discussion}

This secondary analysis sought to explore predictors of response to an outpatient burnout prevention program. Results revealed that participants with higher well-being, better nutritional behavior and lower symptoms of eating disorders experienced lower levels of perceived stress in the follow up. As such, these factors might be considered in a screening procedure when selecting participants for the intervention.

In line with previous behavioral and physiological studies, our study corroborates the association of nutritional behavior including eating disorders and perceived stress. Most individuals under stress experience changes in eating behavior, with approximately $40 \%$ indulging and $40 \%$ under eating. ${ }^{43}$ Stress-induced eating seems to differ based on the type of stressors, with workplace stress having the highest impact followed by ego threatening stress and interpersonal stress bringing out hyperphagic responses, while physical stress elicits hypophagic responses. ${ }^{44,45}$ Independent of whether an individual over or under eats, it is evident that individuals and animals tend to eat highly palatable energy dense food or "comfort food" more than high fiber, low fat, nutrient rich food upon experiencing stress. ${ }^{46-50}$ The promotion 
of food intake during stress has been explained through many biological pathways. Particularly, pathways concerning the release of glucocorticoids by hypothalamic-pituitary-adrenal axis and the activity of brain opioidergic and dopaminergic neurotransmission has been shown to play an important role in alleviating stress and improving mood via the overeating of energy-dense foods with hedonic qualities. ${ }^{51-54}$ This also supports findings that cortisol levels are higher on workdays than weekends. ${ }^{55}$ In addition, micronutrients (essential amino acids, folic acid, tryptophan and vitamins D, B, B6, A and C) and minerals (iron and selenium) appear to have a substantial influence on mood, fatigue and stress experienced by healthy individuals. ${ }^{56-58}$ Taken together, these studies provide striking evidence of the influence of stress on food choice and intake through various biological pathways. Thereby, further solidifying our results which points out that nutritional behavior and symptoms of eating disorders at baseline are strongly associated with the level of perceived stress 6 months after a stress reduction program.

The capability of well-being to predict perceived stress has been consistently explained through research on the Job Demand-Control model (JD-C), ${ }^{59}$ Job Demand-Resource model $(\mathrm{JD}-\mathrm{R})^{60}$ and Effort Reward Imbalance model (ERI). ${ }^{61} \mathrm{JD}-\mathrm{C}$ model attributes low job control and high job demands ("high strain jobs") to be the source of low psychological well-being, ${ }^{62}$ emotional exhaustion, ${ }^{63}$ depression, ${ }^{64}$ neurotic disorder $^{65}$ and increased psychosomatic and physical health complains. ${ }^{63}$ On the other hand, JD-R model posits that high job demands and low job resources impair mental and physical well-being, predict burnout and depreciate workplace motivation. ${ }^{9,66}$ Similarly, employees putting in high psychological and physical efforts but gaining low rewards in terms of salary, support and job security, were found to have lower well-being according to the ERI model. ${ }^{63}$ Overall, our results support the use of wellbeing measures to predict the strength of their response to the stress reduction program in terms of the level of perceived stress. 
Our study reconfirms the importance of a careful participant pre-selection to ensure the effectiveness of interventions in real life settings. The transferability and effectiveness of a program in real life settings does not just depend on its overall effectiveness, but also on the selection of the right program based on the patient's baseline clinical and demographic characteristics - a concept inbuilt in the practice of personalized medicine.$^{67}$ In order to identify these characteristics, studies such as ours that explore pre-treatment variables that predict response and non-response to programs, in terms of high or low levels of the desired outcome, are of significant importance. Our results are in line with similar studies addressing predictors of response to treatment programs of depression, ${ }^{68}$ anxiety disorders ${ }^{69}$ and obsessivecompulsive disorder. ${ }^{70}$ Moreover, a review on psycho-oncological interventions on emotional distress in adult patients with cancer, found studies that selected participants based on baseline distress levels to have the best treatment success rates. ${ }^{71}$ Identifying predictors of response to preselect participants to intervention programs is a systemic strategy that could have a major impact on the successful and sustainable transfer of results of studies evaluating treatment and intervention programs to real life settings, while promoting the effective allocation of resources and providing maximal benefits to patients and health systems.

We find our results on the predictive capabilities of nutritional behavior and eating disorder syndrome to be rather surprising as we expected other variables such as depressive symptoms and stress management to be front runners in predicting perceived stress. However, in the light of available studies and theories focusing on the interrelations of stress, well-being, nutritional behavior and eating disorders, our results seem viable. Moreover, several interventions such as Mindfulness-Based Stress Reduction, Mindfulness-Based Eating Awareness Training and Mindful Eating and Living (MEAL) target stress reduction by increasing awareness of and changing eating behavior, which supports attempts to reduce stress and burnout through changes in nutritional behavior. ${ }^{72,73}$ Because our results emphasize the 
influence of nutrition and eating behavior on perceived stress, perhaps it could be an option to offer such an intervention within or in addition to our burnout prevention program. However, some may argue that poor nutritional behavior and symptoms of eating disorders could simply be symptoms or manifestations of high levels of perceived stress. We agree that we do not know whether poor nutrition behavior are symptoms of stress or whether they are separate yet related issues. We also have no way of clarifying this issue within the boundaries of our study. However, we still find it vital to know whether persons with these symptoms will respond better or worse to the intervention. Hence, it is still important to identify target groups through indicators (such as low well-being, poor nutritional habits and symptoms of eating disorders) in order to provide them with timely and suitable care.

Some limitations need to be considered when interpreting results of this study. While the sample size was sufficient for the original RCT, a larger sample size would have allowed us to consider more predictor variables in the models estimated in this secondary analysis. In addition, the explained variance of the regression model was rather small, which makes us question the existence of other unknown variables that might have contributed to the prediction of perceived stress at last follow up, but were not available in the dataset. Moreover, the results could face selection biases due to a voluntary sample and because the RCT's inclusion criteria might streamline our results to fit a physically "healthy" population with mid to high socioeconomic status, who are insured with specific insurance companies. We also did not expect the significant difference in perceived stress between the Spring and Autumn groups as seen in our core analysis, as the intervention was demonstrated to be effective in the main study. A possible explanation might be a seasonality effect. However, it is important to note that the difference was not significant in our sensitivity analysis. In retrospect, the analysis should have taken in to account factors such as number of hours worked, being employed in multiple jobs, marital status and the number and age of dependents, as these factors have a 
direct impact on stress levels. The study may also pose limited external validity as it is specific to Germany. A longer follow-up (more than 6 months) would have permitted us to better characterize the results' sustainability. However, the original study was faced with time and funding constraints which limited their ability to extend the follow-up period. Lastly, clinical characteristics were evaluated using self-reported measures which come with its potential biases influenced by personal traits and situational factors. Strengths of this study include the use of validated assessment tools, the control and exclusion of confounders in regression modelling and the consistency of most results with the sensitivity analysis despite the use of completely different imputation methods.

\section{Conclusion}

In conclusion, the burnout prevention program may not be suitable for individuals with lower levels of well-being, poor nutritional behavior and an elevated number of symptoms related to eating disorders, as they appear to be a group with considerable psychiatric distress. Potential participants with low well-being, poor nutritional behaviors and symptoms of eating disorders, could be referred to more appropriate and intensive interventions or provided with additional care whilst enrolled in the burnout prevention program. Therefore, burnout prevention programs might require more rigorous screening interviews. This may contribute to enhanced effectiveness of such programs in real-life settings. Thus, the results of this study can serve as guidance for health care professionals and health insurance providers when recommending their clients to the program, thereby promoting the effective allocation of financial resources and maximizing individual benefits to the employee in terms of stress reduction and burnout prevention. 


\section{Reference}

1. Black D. Working for a healthier tomorrow. UK: Cross-Goverment Health. Work and Well-being Programme. 2008.

2. Harvey SB, Henderson M, Lelliott P, Hotopf M. Mental health and employment: much work still to be done. RCP; 2009.

3. Whiteford HA, Degenhardt L, Rehm J, et al. Global burden of disease attributable to mental and substance use disorders: findings from the Global Burden of Disease Study 2010. The Lancet. 2013;382(9904):1575-1586.

4. Badura B, Ducki A, Schröder H, Klose J, Macco K. Fehlzeiten-Report 2011. Schwerpunktthema: Führung und Gesundheit. Berlin. 2011.

5. Kaschka WP, Korczak D, Broich K. Burnout: a fashionable diagnosis. Deutsches Ärzteblatt International. 2011;108(46):781.

6. Stier-Jarmer M, Frisch D, Oberhauser C, Berberich G, Schuh A. The Effectiveness of a Stress Reduction and Burnout Prevention Program: A Randomized Controlled Trial of an Outpatient Intervention in a Health Resort Setting. Deutsches Ärzteblatt International. 2016;113(46):781.

7. Colligan TW, Higgins EM. Workplace stress: Etiology and consequences. Journal of workplace behavioral health. 2006;21(2):89-97.

8. Bianchi R, Schonfeld IS, Laurent E. Burnout-depression overlap: A review. Clinical psychology review. 2015;36:28-41.

9. Schaufeli WB, Leiter MP, Maslach C. Burnout: 35 years of research and practice. Career development international. 2009;14(3):204-220.

10. Korczak D, Wastian M, Schneider M. Therapie des Burnout-Syndroms. Schriftenreihe Health Technology Assessment (HTA) in der Bundesrepublik Deutschland (Bd 120). Deutsches Institut für Medizinische Dokumentation und Information (DIMDI), Köln. 2012.

11. Toppinen-Tanner S, Ojajarvi A, Vaananen A, Kalimo R, Jappinen P. Burnout as a predictor of medically certified sick-leave absences and their diagnosed causes. Behav Med. Spring 2005;31(1):18-27.

12. Hemingway H, Marmot M. Clinical Evidence: Psychosocial factors in the etiology and prognosis of coronary heart disease: systematic review of prospective cohort studies. Western Journal of Medicine. 1999;171(5-6):342.

13. Melamed S, Kushnir T, Shirom A. Burnout and risk factors for cardiovascular diseases. Behav Med. Summer 1992;18(2):53-60.

14. Antoni MH, Lutgendorf SK, Cole SW, et al. The influence of bio-behavioural factors on tumour biology: pathways and mechanisms. Nature reviews. Cancer. 2006;6(3):240.

15. Hu FB, Meigs JB, Li TY, Rifai N, Manson JE. Inflammatory markers and risk of developing type 2 diabetes in women. Diabetes. 2004;53(3):693-700.

16. Finestone HM, Alfeeli A, Fisher WA. Stress-induced physiologic changes as a basis for the biopsychosocial model of chronic musculoskeletal pain: a new theory? The Clinical journal of pain. 2008;24(9):767-775.

17. Kivimaki M, Leino-Arjas P, Virtanen M, et al. Work stress and incidence of newly diagnosed fibromyalgia: prospective cohort study. Journal of psychosomatic research. 2004;57(5):417-422.

18. Larzelere MM, Jones GN. Stress and health. Primary Care: Clinics in Office Practice. 2008;35(4):839-856. 
19. Michie S, Williams S. Reducing work related psychological ill health and sickness absence: a systematic literature review. Occupational and environmental medicine. 2003;60(1):3-9.

20. Jennings BM. Work stress and burnout among nurses: Role of the work environment and working conditions. 2008.

21. Limm H, Gündel H, Heinmüller M, et al. Stress management interventions in the workplace improve stress reactivity: a randomised controlled trial. Occupational and environmental medicine. 2010:oem. 2009.054148.

22. Erickson R, Grove W. Why emotions matter: age, agitation, and burnout among registered nurses. Online journal of issues in nursing. 2007;13(1):1-13.

23. Gilboa S, Shirom A, Fried Y, Cooper C. A meta-analysis of work demand stressors and job performance: examining main and moderating effects. Personnel Psychology. 2008;61(2):227-271.

24. Zangaro GA, Soeken KL. A meta-analysis of studies of nurses' job satisfaction. Research in nursing \& health. 2007;30(4):445-458.

25. Kammer BP. BPtK-Studie zur Arbeitsunfähigkeit. Psychische Erkrankungen und Burnout, Berlin. 2012.

26. Hinding B, Kastner M. Gestaltung von lernförderlichen Unternehmenskulturen zu Sicherheit und Gesundheit bei der Arbeit. Bundesanstalt für Arbeitsschutz und Arbeitsmedizin (BAuA), Dortmund. 2011.

27. für Arbeitsschutz B. Arbeitsmedizin (BAuA)(2015) Volkswirtschaftliche Kosten durch Arbeitsunfähigkeit 2013. 2013.

28. Bödeker W, Friedrichs M. Kosten der psychischen Erkrankungen und Belastungen in Deutschland. Regelungslücke psychische Belastungen schliessen, Hans Bockler Stiftung (L. Kamp and K. Pickshaus, eds.), Dusseldorf. 2011:69-102.

29. Giga SI, Noblet AJ, Faragher B, Cooper CL. The UK perspective: A review of research on organisational stress management interventions. Australian Psychologist. 2003;38(2):158-164.

30. Graveling R, Crawford J, Cowie H, Amati C, Vohra S. A review of workplace interventions that promote mental wellbeing in the workplace. Edinburgh: Institute of Occupational Medicine. 2008.

31. Van der Klink J, Blonk R, Schene AH, Van Dijk F. The benefits of interventions for work-related stress. American journal of public health. 2001;91(2):270.

32. Schaufeli WB. Maslach burnout inventory-general survey (MBI-GS). MBI manual. 1996:19-26.

33. Fliege H, Rose M, Arck P, Levenstein S, Klapp B. Validierung des "perceived stress questionnaire"(PSQ) an einer deutschen stichprobe. Diagnostica. 2001;47(3):142152.

34. Fliege H, Rose M, Arck P, et al. The Perceived Stress Questionnaire (PSQ) reconsidered: validation and reference values from different clinical and healthy adult samples. Psychosomatic medicine. 2005;67(1):78-88.

35. Maslach C, Jackson SE, Leiter MP. Maslach burnout inventory. CPP; 2006.

36. Arnold S. Kohärenzgefühl und Burnout bei Hochbegabten-eine Querschnittstudie.

37. J G. Personal communication per email on 12.06.2013. 2013.

38. Tritt K, von Heymann F, Zaudig M, Zacharias I, Söllner W, Loew T. Development of the" ICD-10-Symptom-Rating"(ISR) questionnaire. Zeitschrift fur Psychosomatische Medizin und Psychotherapie. 2008;54(4):409-418.

39. Tritt K, von Heymann F, Zaudig M, et al. The ICD-10-Symptom-Rating (ISR) - Brief Description. In: Institute for Quality Development in Psychotherapy and Psychosomatics M, Germany, ed2013. 
40. Anwenderhandbuch Evaluation Teil 1:Evaluation des Individuellen Ansatzes Kursmassnahmen in den Handlungsfeldern Bewegungsgewohnheiten, Ernaehrung und Stressmanagement. . In: (Hrsg.) G-S, ed. Berlin2014.

41. Dong Y, Peng C-YJ. Principled missing data methods for researchers. SpringerPlus. 2013;2(1):222.

42. Rezvan PH, Lee KJ, Simpson JA. The rise of multiple imputation: a review of the reporting and implementation of the method in medical research. BMC medical research methodology. 2015;15(1):30.

43. James PT, Leach R, Kalamara E, Shayeghi M. The worldwide obesity epidemic. Obesity. 2001;9(S11).

44. O'connor DB, Jones F, Conner M, McMillan B, Ferguson E. Effects of daily hassles and eating style on eating behavior. Health Psychology. 2008;27(1):S20.

45. Newman E, O'Connor DB, Conner M. Daily hassles and eating behaviour: the role of cortisol reactivity status. Psychoneuroendocrinology. 2007;32(2):125-132.

46. Oliver G, Wardle J, Gibson EL. Stress and food choice: a laboratory study. Psychosomatic medicine. 2000;62(6):853-865.

47. Zellner DA, Loaiza S, Gonzalez Z, et al. Food selection changes under stress. Physiology \& behavior. 2006;87(4):789-793.

48. Wallis DJ, Hetherington MM. Emotions and eating. Self-reported and experimentally induced changes in food intake under stress. Appetite. 2009;52(2):355-362.

49. Dallman MF, Pecoraro NC, La Fleur SE, et al. Glucocorticoids, chronic stress, and obesity. Progress in brain research. 2006;153:75-105.

50. Pecoraro N, Reyes F, Gomez F, Bhargava A, Dallman MF. Chronic stress promotes palatable feeding, which reduces signs of stress: feedforward and feedback effects of chronic stress. Endocrinology. 2004;145(8):3754-3762.

51. Gibson EL. Emotional influences on food choice: sensory, physiological and psychological pathways. Physiology \& behavior. 2006;89(1):53-61.

52. Born JM, Lemmens SG, Rutters F, et al. Acute stress and food-related reward activation in the brain during food choice during eating in the absence of hunger. International journal of obesity. 2010;34(1):172.

53. Hagan MM, Chandler PC, Wauford PK, Rybak RJ, Oswald KD. The role of palatable food and hunger as trigger factors in an animal model of stress induced binge eating. international Journal of Eating disorders. 2003;34(2):183-197.

54. Tomiyama AJ, Dallman MF, Epel ES. Comfort food is comforting to those most stressed: evidence of the chronic stress response network in high stress women. Psychoneuroendocrinology. 2011;36(10):1513-1519.

55. Kunz-Ebrecht SR, Kirschbaum C, Marmot M, Steptoe A. Differences in cortisol awakening response on work days and weekends in women and men from the Whitehall II cohort. Psychoneuroendocrinology. 2004;29(4):516-528.

56. Hakkarainen R, Partonen T, Haukka J, Virtamo J, Albanes D, Lönnqvist J. Food and nutrient intake in relation to mental wellbeing. Nutrition Journal. 2004;3(1):14.

57. Laugero KD, Falcon LM, Tucker KL. Relationship between perceived stress and dietary and activity patterns in older adults participating in the Boston Puerto Rican Health Study. Appetite. 2011;56(1):194-204.

58. Long S-J, Benton D. Effects of vitamin and mineral supplementation on stress, mild psychiatric symptoms, and mood in nonclinical samples: a meta-analysis. Psychosomatic medicine. 2013;75(2):144-153.

59. Karasek Jr RA. Job demands, job decision latitude, and mental strain: Implications for job redesign. Administrative science quarterly. 1979:285-308. 
60. Demerouti E, Bakker AB, Nachreiner F, Schaufeli WB. The job demands-resources model of burnout. Journal of Applied psychology. 2001;86(3):499.

61. Siegrist J. Adverse health effects of high-effort/low-reward conditions. Journal of occupational health psychology. 1996;1(1):27.

62. Häusser JA, Mojzisch A, Niesel M, Schulz-Hardt S. Ten years on: A review of recent research on the Job Demand-Control (-Support) model and psychological well-being. Work \& Stress. 2010;24(1):1-35.

63. de Jonge J, Bosma H, Peter R, Siegrist J. Job strain, effort-reward imbalance and employee well-being: a large-scale cross-sectional study. Social science \& medicine. 2000;50(9):1317-1327.

64. Mausner-Dorsch H, Eaton WW. Psychosocial work environment and depression: epidemiologic assessment of the demand-control model. American journal of public health. 2000;90(11):1765.

65. Cropley M, Steptoe A, Joekes K. Job strain and psychiatric morbidity. Psychological medicine. 1999;29(6):1411-1416.

66. Bakker AB, Demerouti E, Verbeke W. Using the job demands-resources model to predict burnout and performance. Human resource management. 2004;43(1):83-104.

67. Wolitzky-Taylor KB, Arch JJ, Rosenfield D, Craske MG. Moderators and nonspecific predictors of treatment outcome for anxiety disorders: A comparison of cognitive behavioral therapy to acceptance and commitment therapy. Journal of Consulting and Clinical Psychology. 2012;80(5):786.

68. Simon GE, Perlis RH. Personalized medicine for depression: can we match patients with treatments? American Journal of Psychiatry. 2010;167(12):1445-1455.

69. Meuret AE, Rosenfield D, Seidel A, Bhaskara L, Hofmann SG. Respiratory and cognitive mediators of treatment change in panic disorder: Evidence for intervention specificity. Journal of consulting and clinical psychology. 2010;78(5):691.

70. Maher M, Huppert JD, Chen H, et al. Moderators and predictors of response to cognitive-behavioral therapy augmentation of pharmacotherapy in obsessivecompulsive disorder. Psychological medicine. 2010;40(12):2013-2023.

71. Faller H, Schuler M, Richard M, Heckl U, Weis J, Küffner R. Effects of psychooncologic interventions on emotional distress and quality of life in adult patients with cancer: systematic review and meta-analysis. Journal of Clinical Oncology. 2013;31(6):782-793.

72. Daubenmier J, Kristeller J, Hecht FM, et al. Mindfulness intervention for stress eating to reduce cortisol and abdominal fat among overweight and obese women: an exploratory randomized controlled study. Journal of obesity. 2011;2011.

73. Dalen J, Smith BW, Shelley BM, Sloan AL, Leahigh L, Begay D. Pilot study: Mindful Eating and Living (MEAL): weight, eating behavior, and psychological outcomes associated with a mindfulness-based intervention for people with obesity. Complementary therapies in medicine. 2010;18(6):260-264. 